\title{
ABSTRACTS OF SELECTED PAPERS
}

Traumatic lesions of the cervical spine (a survey of 430 cases), by Prof. José C. de Oliveira. Rev. Ortop. Traum., (1980), 6P IB, 2: I I 5-I45.

Clinical signs of hyperextension injuries must be looked for as radiography can be inconclusive.

Some cases of horizontal shearing fractures, so far not described in the cervical spine, are shown.

Out of 82 compression fractures, 2I were of cuneiform and 6I of tear-drop type. Anterior fusion may be necessary to prevent kyphosis. Tear-drop fractures are among the most severe cervical injuries. Anterior partial corporectomy is indicated when skull traction does not reduce compression of the posterior part of the vertebral body.

\section{SARRIAS}

On the pathogenesis of bedsores. Skin blood cessation by external pressure on the back, by B. Lassen, P. Holstein \& N. A. Lassen. Scandinavian fournal of Plastic \& Reconstructive Surgery, (1979), 13, 347.

This was an experimental study designed to measure the amount of pressure required to produce cessation of skin blood flow in humans lying supine. 'Flow cessation external pressure (FCEP) was measured using the washout of an intracutaneous depot of $\mathrm{I}_{131}$ antipyrine mixed with histamine. The FCEP was closely correlated to the mean blood pressure in normals, patients with hypertension and patients with paraplegia or tetraplegia. The difference between mean blood pressure less FCEP averaged $4 \mathrm{~mm}$. Hg., all three groups behaving in a similar fashion. As expected, the conclusion was that external pressure exceeding the mean blood pressure led to cessation of skin circulation.

D. O. MAISELS

\section{The biceps femoris musculocutaneous flap in the repair of pressure sores around} the hip, by J. H. James and I. H. Moir. Plastic \& Reconstructive Surgery, (I980), 66, 736 .

An island of skin carried on the long head of biceps femoris has been used in several cases for the repair of ischial and trochanteric sores. There were no cases of flap necrosis and healing was satisfactory.

D. O. MAISELS

Differential response of skin and muscle in the experimental production of pressure sores, by G. T. Nola and L. M. Vistres. Plastic \& Reconstructive Surgery, (1980), 66, 728.

An experimental model was designed for the production of pressure sores over a bony prominence. While the interposition of a muscle flap between the skin and subcutaneous tissues and the bone reduced the incidence of skin ulceration, there was, nevertheless, histological evidence of muscle necrosis in the absence of any changes in the skin. The obvious conclusion is that muscle is more susceptible to pressure necrosis than skin and subcutaneous tissues and thus not the ideal padding material.

D. O. MAISELS 
Primary closure of trochanteric decubitous ulcers: The bipedicled tensor fascia lata musculocutaneous flap, by N. H. Schulman. Plastic \& Reconstructive Surgery, (1980), 66, 740.

The use of a bipedicled tensor fascia lata myocutaneous flap is described for the repair of trochanteric sores. The donor area is closed by direct suture.

D. O. MAISELS

Paraplegia due to epidural anaesthesia, by G. A. Zach, W. Kuhn and A. Urwyler, Swiss Paraplegic Centre, Basle, Switzerland.

The possible occurrence of paraplegia arising as a complication following epidural anaesthesia, was discussed. Lumbar epidural anaesthesia can be used for all types of surgery below the umbilicus, for sympathetic block, for obstetric analgesia and such specific problems of paraplegia as spasticity, pain and bladder complications. Thoracic epidural anaesthesia is used mainly for pain relief and improvement of spontaneous ventilation in severe chest injuries.

Three patients who developed paraplegia were discussed. The first probably resulted from an extensive epidural haematoma, the second to a paravertebral abscess, and the third may have been caused by a combination of vascular hypotension and infection.

In the first two patients myelography revealed a hold-up, so that laminectomy was carried out.

However, in all three patients the final result was disappointing, with considerable permanent neurological deficit. 